SAR records. The resulting data were cross-referenced with ED records.

Results.-In all, 789 casualties were rescued during the study period. Both prehospital and hospital records from all applicable sources were available for $60 \%$, with full ED records available for $94 \%$. Involvement of the SAR helicopter was documented in 569 cases (72\%), and of MRT in 733 cases (93\%). There were 70 fatalities, including 5 pediatric fatalities. More than a third were attributed to medical causes. Nearly two-thirds $(63 \%)$ of all casualties and $93 \%$ of fatalities were male. Eighty-seven casualties $(11 \%)$ were under age 18 ; onethird of casualties were aged 28 years or less, one-third were aged 29 to 49 years, and the remainder were aged 50 years or less. The 2 youngest casualties were both aged 3 years, and the oldest was 86 years. More than two-thirds of the casualties had been hill walking. Most of the remainder were rock climbing $(11 \%)$ or scrambling $(7 \%)$. Overall, only $19 \%$ of casualties were medical in nature, whereas the vast majority were the result of trauma. Of the injuries, more than half (55\%) were lower limb injuries.

Conclusions.-Nearly 100 patients each year, of all ages, are managed in our ED after being assisted by our MRT and SAR colleagues. Most are hill walkers, and the majority are injured rather than ill, but nearly one-fifth of mountain casualties present with medical problems.

Benjamin G. R. Hall, MBChB Linda K. Dykes, MBBS (Hons), FCEM Bangor, North Wales, UK

\section{A Prospective Analysis of Lifeguard Medical Team Interventions in Ecuador During 2011 Carnaval}

Background.-Drowning remains a leading cause of death globally. The true burden is unknown because of underreporting. Carnaval, a large-scale event in Ecuador, draws large sections of the population to the coast and historically is associated with numerous drowning deaths.

Objective.-The purpose of this study was to prospectively quantify preventative, rescue, and first aid interventions by lifeguards protecting the beaches in Ecuador during Carnaval.

Methods.-This was an Institutional Review Board approved, prospective observational study. Inclusion criteria were any rescue, prevention, or first aid encounters by lifeguards. Lifeguards were trained on the 18-item data collection form and dictionary, and completed the form after every intervention. Elements collected included age, gender, time of day, injuries, contributing factors, disposition, and mortality. The primary measure was the proportion of the 3 types of interventions. We secondarily characterize the lifeguard rescues. Data were entered into a database by trained and monitored research assistants. We report descriptive statistics as appropriate.

Results.-During the 4-day event, lifeguards performed 246 unique interventions: 153 preventative maneuvers on 2141 swimmers, 94 water rescues, and 29 first aid interventions. The mean age was 22.7 years (range 3-60 years). The rescued swimmers were predominantly male, 36 of $52(69 \%$ [95\% confidence interval (confidence interval): $55 \%$ to $80 \%$ ]). The time of day analysis revealed a bimodal pattern with peaks during the 11:00 AM to 12:00 noon and the 1:00 PM to 2:00 PM periods. Rip current was a factor in 52 of 94 cases $(55 \%$ [95\% CI: $45 \%$ to $65 \%$ ]), and alcohol was a factor in 4 of 47 rescues (9\% [95\% CI: $3 \%$ to $21 \%]$ ). None of the 94 rescues (0\% [95\% CI: $0 \%$ to $5 \%$ ]) required resuscitation, and only 2 of $94(2 \%$ [95\% CI: $1 \%$ to $7 \%$ ]) required transfer to a hospital. There were no drowning deaths. Study limitations were that beach crowd estimates and number of persons impacted by preventative interventions were not adjudicated.

Conclusions.-The majority of lifeguard interventions were preventative in nature, and there were no drowning deaths. Most rescues occurred in the late morning or early afternoon, and involved male, nonintoxicated swimmers. Our data may provide insight for others planning large water events in the future.

Justin R Sempsrott, MD

Allie Moriarty, BS

David Slattery, MD

Las Vegas, NV, USA

Andrew Schmidt, DO

Jacksonville, FL, USA

\section{Intraocular Pressure During Acute Exposure to High Altitude}

Background.-High altitude exposure is increasing owing to the constantly rising numbers of mountaineers and trekkers, and it is a clinically relevant cause of complex pathophysiological events leading to alterations in all parts of the body, including the visual system. Previous reports on intraocular pressure (IOP) changes during high altitude exposure have provided inconsistent and even conflicting results.

Objective.-The purpose of our study was to investigate the effect of acute high altitude exposure on IOP using state-ofthe-art Goldmann applanation tonometry and to assess a correlation with AMS and vital parameters.

Methods.-Fourteen healthy lowlanders ascended from Tübingen, Germany (341 m), to the Capanna Margherita, Italy (4559 m), during the Tübingen High Altitude Ophthalmology research study within 24 hours, including 1 overnight stay at $3647 \mathrm{~m}$. The IOP measurements using a Goldmann tonometer (Haag-Streit, Switzerland) and AMS scores (Lake Louise and AMS-c scores) were assessed twice daily; oxygen saturation $\left(\mathrm{SpO}_{2}\right)$ and heart rate were monitored daily. Baseline recordings were taken before and at least 2 weeks after high altitude exposure at the University Eye Hospital in Tübingen. Intraindividual differences of IOP were calculated using multivariate analysis of variance and $95 \% \mathrm{CI}$.

Results.-The IOP was decreased on day 2 by an average of $-0.57 \mathrm{~mm} \mathrm{Hg}$ (mean intraindividual difference; 95\% CI: -1.71 to $0.56 \mathrm{~mm} \mathrm{Hg}$ ) and remained decreased during high altitude exposure; all values were statistically not significant compared with baseline. Incidence of AMS according to AMS scores was $50 \%$ on day 2. Subjects with AMS had lower $\mathrm{SpO}_{2}$ $(71.70 \% \pm 6.54 \%)$ and higher heart rate $(85.70 \pm 9.00$ beats/ 